\title{
BI.INTEGRATION METHODOLOGIES FOR RISK MANAGEMENT IN THE BIOPHARMACEUTICAL INDUSTRY
}

\author{
Miguel Angel de la O Herrera ${ }^{1}$; Elezer Monte Blanco Lemes ${ }^{1}$. \\ ${ }^{1}$ Bio-Manguinhos / Fiocruz.
}

INTRODUCTION The biopharmaceutical industry has been working recently to attend the suggestion of international standards to structure a Risk Assessment Quality System. For example, the ICH (International Conference on Harmonization) through the Q9 guide, recommends the use of risk analysis tools to support the risk management process. However, methodologies for identification and analysis of failures and deviations proposed in this guide are focused mainly for risk management at specific targets, such as processes, noncompliance, equipment, infrastructure, etc., and is commonly necessary to apply independently more than one tool for analysis and identification of risk.

OBJECTIVE To propose a Hazard and Operability Study (HAZOP) and the Failure Modes and Effects Analysis (FMEA) integrated methodology for risk analysis, which allows the identification and treatment of deviations in equipment and new infrastructure within the biopharmaceutical industry, from the analysis of operating parameters and unit operations used in production processes.

METHODOLOGY To perform this work, a process for recombinant protein production was analyzed as a case of study. Key unit operations comprising process stages were characterized. For each process, parameter causes and consequences of possible deviations were defined; subsequently the level of risk was established, as indicated by HAZOP methodology. Likewise, detection measures and controls were identified; finally, based on the concepts of the FMEA methodology, the Risk Prioritization Number (RPN) was calculated.

RESULTS In the multiplication stages 4 nodes were identified with 15 possible deviations, being $90 \%$ considered undesirable and $10 \%$ acceptable. The bioreaction stage showed 33 deviations divided in 7 nodes, being 21\% acceptable and 79\% undesirable. The culture centrifugation and inactivation stage had 5 nodes with 33 deviations, $51 \%$ acceptable and $49 \%$ undesirable. In the cell disruption stage 28 deviations 
were identified in 6 nodes. 21\% of deviations were acceptable, $71 \%$ undesirable and $8 \%$ unacceptable. Finally, in the washing of inclusion bodies stages 35 deviations in 6 nodes were found, being 31\% acceptable and 69\% undesirable. Considering the high incidence of deviations cataloged as undesirable, the concept of detectability of deviations was applied. Detection rates allowed establishing the RPN, thus facilitating the prioritization of deviations treatment regardless of their level of risk.

CONCLUSION The proposed methodology allowed the simultaneous analysis of deviations in operating parameters and the failure modes of process functions, bringing benefits such as the sparing of resources and required time to implement a risk management program. Prioritization in the treatment of risks was used in structuring a plan of action to mitigate the risks.

KEYWORDS HAZOP, FMEA, risk. 